\title{
Biomechanical factors in planning of periacetabular osteotomy
}

\author{
Noushin Niknafs ${ }^{1}$, Ryan J. Murphy ${ }^{2,3}$, Robert S. Armiger ${ }^{2}$, Jyri Lepistö $^{4}$ and Mehran Armand ${ }^{2,3 *}$ \\ 1 Department of Biomedical Engineering, Johns Hopkins University, Baltimore, MD, USA \\ ${ }^{2}$ Department of Research and Engineering Development, Johns Hopkins University Applied Physics Laboratory, Laurel, MD, USA \\ ${ }^{3}$ Department of Mechanical Engineering, Johns Hopkins University, Baltimore, MD, USA \\ ${ }^{4}$ ORTON Orthopaedic Hospital, Helsinki, Finland
}

\section{Edited by:}

Tarun Goswami, Wright State

University, USA

Reviewed by:

Xiaogai Li, Royal Institute of

Technology, Sweden

Andreas Leithner, Medical University

of Graz, Austria

${ }^{*}$ Correspondence:

Mehran Armand, Johns Hopkins

University Applied Physics Laboratory,

11100 Johns Hopkins Road, 21-S185,

Laurel, MD 20723, USA

e-mail: mehran.armand@jhuapl.edu
Objective: This study addresses the effects of cartilage thickness distribution and compressive properties in the context of optimal alignment planning for periacetabular osteotomy (PAO).

Background: The Biomechanical Guidance System (BGS) is a computer-assisted surgical suite assisting surgeon's in determining the most beneficial new alignment of a patient's acetabulum. The BGS uses biomechanical analysis of the hip to find this optimal alignment. Articular cartilage is an essential component of this analysis and its physical properties can affect contact pressure outcomes.

Methods: Patient-specific hip joint models created from CT scans of a cohort of 29 dysplastic subjects were tested with four different cartilage thickness profiles (one uniform and three non-uniform) and two sets of compressive characteristics. For each combination of thickness distribution and compressive properties, the optimal alignment of the acetabulum was found; the resultant geometric and biomechanical characterization of the hip were compared among the optimal alignments.

Results: There was an average decrease of $49.2 \pm 22.27 \%$ in peak contact pressure from the preoperative to the optimal alignment over all patients. We observed an average increase of $19 \pm 7.7^{\circ}$ in center-edge angle and an average decrease of $19.5 \pm 8.4^{\circ}$ in acetabular index angle from the preoperative case to the optimized plan. The optimal alignment increased the lateral coverage of the femoral head and decreased the obliqueness of the acetabular roof in all patients. These anatomical observations were independent of the choice for either cartilage thickness profile, or compressive properties.

Conclusion: While patient-specific acetabular morphology is essential for surgeons in planning PAO, the predicted optimal alignment of the acetabulum was not significantly sensitive to the choice of cartilage thickness distribution over the acetabulum. However, in all groups the biomechanically predicted optimal alignment resulted in decreased joint contact pressure and improved acetabular coverage.

Keywords: periacetabular osteotomy, preoperative planning, articular cartilage thickness, cartilage compressibility, biomechanical analysis

\section{INTRODUCTION}

Numerous outcome studies performed during the last 30 years have shown that performing periacetabular osteotomy (PAO) on young adults with dysplasia is a very effective surgery and prevents or delays osteoarthritis of the hip (Siebenrock et al., 1999; Trumble et al., 1999; Ganz and Leunig, 2007). Typically, surgeons plan the osteotomy on the basis of geometry so that the radiological angles, representing contact surface orientation, reproduce those of normal hips (Wiberg, 1939; Anda et al., 1991; Tallroth and Lepisto, 2006). Several authors (e.g., Hipp et al., 1999; Chao et al., 2000; Mechlenburg et al., 2010; Zhao et al., 2010) have shown that biomechanical planning based on hip geometry corresponds to what surgeons do in practice. However, none of the literature review how the inclusion of biomechanical parameters (especially cartilage thickness) may further affect both planning and the surgical outcome.

The Biomechanical Guidance System (BGS) (Armand et al., 2004, 2005a; Armiger, 2006; Lepistö et al., 2008; Armiger et al., 2009) is a computer-assisted surgical suite for performing PAO. The system combines geometric and biomechanical feedback with intra-operative tracking to guide the surgeon through the $\mathrm{PAO}$ procedure. The BGS performs discrete element analysis (DEA) to estimate the contact pressure on a patient-specific model of the joint surface (An et al., 1990). DEA has been shown to approximate the location and magnitude of the peak contact pressure with an accuracy that is not significantly different from that of 
more computationally expensive finite element methods (Li et al., 1997) in much shorter periods of time, though the distribution is smoother and peak pressures are underestimated.

During contact pressure analysis with DEA, one can consider a variety of biomechanical factors. Choices for modeling the variation of cartilage compressibility determine the DEA type (linear vs. non-linear), and choices for cartilage thickness distribution affect the stiffness matrix computation. Cartilage thickness distribution has been the subject of several studies (Rushfeldta et al., 1981; Hodler et al., 1992; Athanasiou et al., 1994; Nishii et al., 2004, 2005) which have measured thickness maps for acetabular and femoral cartilage over the joint contact surface in normal and dysplastic patients. Nishii et al. (2004) conducted an analysis of magnetic resonance (MR) scans from normal and dysplastic cohorts to define thickness distribution of acetabular cartilage and noted the significant differences between the two cohorts. Several studies have reported on the significance of subject-specific acetabular and femoral geometries (e.g., Anderson et al., 2008, 2010; Lenaerts et al., 2008, 2009; Chegini et al., 2009; Gu et al., 2010), and cartilage thickness and distribution (e.g., Anderson et al., 2008, 2010) to the contact pressures found in the hip joint. Despite the evidence for the significance of subject-specific cartilage thickness variations, these variations have not - to our knowledge - been incorporated into the biomechanical analysis in computerized planning of $\mathrm{PAO}$ (Hipp et al., 1999; Armand et al., 2004, 2005b; Armiger et al., 2009).

Several studies (e.g., Abraham et al., 2013) have indicated that loading the hip joint can, in certain circumstances, result in multiple peaks. Based on its formulation, DEA will produce only a single peak in response to a loading profile. However, to our knowledge, no studies have investigated how the single peak produced from DEA impacts biomechanical planning for PAO through correlation with surgical practice.

In this study, we investigate the potential differences between the biomechanically predicted optimal alignment for the acetabular fragment of dysplastic hips under varying cartilage thickness and compressibility models using patient-specific acetabular geometries derived from preoperative CT scans. Specifically: would varying cartilage thickness or the cartilage compressibility model in defining the optimal biomechanical alignment of the hip significantly affect surgeons' current practice and change the alignment goals of PAO (Hipp et al., 1999; Armand et al., 2004, 2005b; Armiger et al., 2009).

\section{MATERIALS AND METHODS}

This study was a retrospective evaluation of preoperative CT scans of a cohort of 29 patients ( 26 female, 3 male) treated with PAO (13 left hip, 16 right hip) under Institutional Review Board approval (JHM IRB1 \#05-09-02-01). All patients were diagnosed with developmental dysplasia of the hip and underwent PAO at Orton Hospital in Helsinki, Finland. Nineteen patients were treated between October 1995 and February 1997; the remaining 10 were treated between November 2005 and April 2006. Patients with concurrent pathologies of slipped capital femoral epiphysis and Legg-CalvéPerthes syndrome were excluded from both $\mathrm{PAO}$ and the present study.

Each patient scan was performed in the supine orientation and covered the entire joint region (the acetabulum and proximal femur) in axial slices. Axial slices comprising the scanned volume had variable and sometimes non-uniform spacing; however, the maximum slice spacing was smaller than $1.6 \mathrm{~mm}$ in the entire scan volumes and the hip joint was usually scanned at higher resolution compared to the rest of the scan volume. For consistency, the scan volumes were re-sampled at $1 \mathrm{~mm}$ spacing and realigned such that the $X$ axis contained the centers of the femoral heads (Figure 1).

\subsection{JOINT MODEL CREATION}

Since bone morphology has been shown to play a significant role in prediction of cartilage stress (Anderson et al., 2008, 2010; Lenaerts et al., 2008, 2009; Chegini et al., 2009; Gu et al., 2010), we manually extracted subject-specific surface models of the femoral head and acetabulum. These subject-specific, nonspherical surface models were created using the Lunate-Trace algorithm (Armiger et al., 2007), which rotates oblique CT reformats of the hip joint about the medio-lateral axis of the hip (Figure 2). Using this procedure, the acetabular and femoral
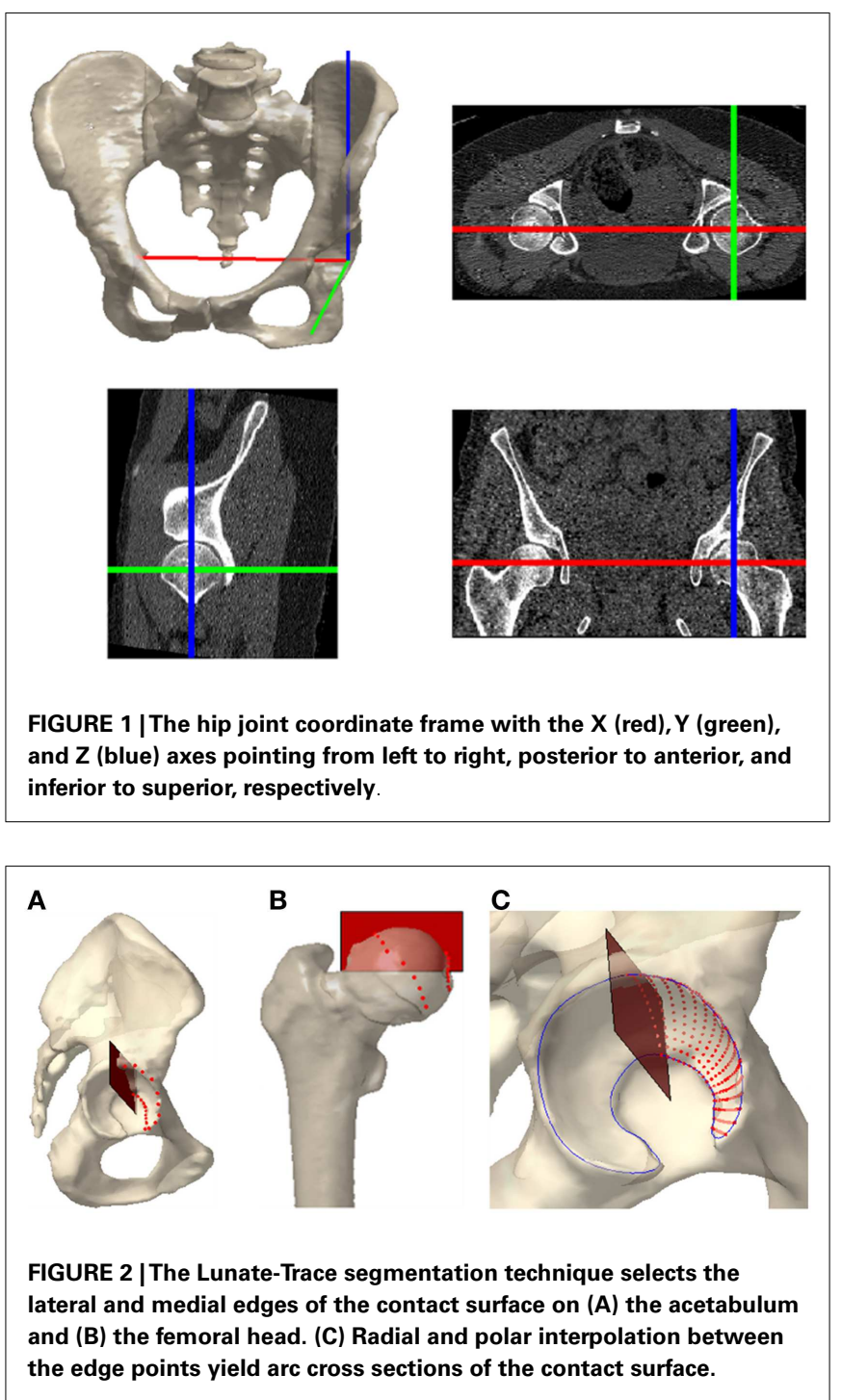
surfaces were specified as triangular surface meshes. The acetabular surface meshes were composed of a set of $1648 \pm 150$ triangular elements, each $1.49 \mathrm{~mm} \pm 0.74 \mathrm{~mm}$ in area. In previous (unpublished) analysis on the Lunate-Trace algorithm, there was an average area difference of $185.6 \mathrm{~mm} \pm 154.5 \mathrm{~mm}$ in the acetabular meshes between two trained users corresponding to an average difference in contact pressures of $0.027 \pm 0.24 \mathrm{MPa}$, indicating minor variability in biomechanical analysis between independently segmented acetabulums. The surface meshes used in the present study were generated by a trained user.

\subsection{CARTILAGE REPRESENTATION}

We created four distinct cartilage profiles for each contact surface: a uniform thickness profile, population-based normal and dysplastic profiles, and a CT-based profile. All generated acetabular surface models were subject-specific. However, one can assume various models for cartilage thickness distribution over a given acetabular surface. The first profile was a uniformly distributed layer of cartilage, where the biomechanics are independent of the arbitrary thickness value of $2.66 \mathrm{~mm}$ (Rushfeldta et al., 1981) used in the simulation.

The second and third thickness maps were based on the mean cartilage distribution profile in normal and dysplastic populations (Nishii et al., 2004). The average distribution maps were expressed as the mean cartilage thickness measured over a grid of longitude and latitude across the weight-bearing area (Figure 3). We conducted a two-dimensional Gaussian fit to these data to define models corresponding to the average cartilage thickness maps in dysplastic and normal populations. We aligned the patient-specific acetabular contact surface in a spherical coordinate frame consistent with the Gaussian model. Note that this was not a spherical fit, as the geometries of the dysplastic hips are not spherical. Then, we applied the Gaussian model to the manually segmented contact surface to derive population-based cartilage thickness maps for each patient. Despite the dysplastic nature of the hips in this study, we included both normal and dysplastic population-based cartilage thickness models since recent research has suggested that PAO helps to normalize force distribution (Mechlenburg et al., 2010).

The fourth thickness map was an estimate of the subjectspecific cartilage thickness. Here, we took the femoral and acetabular contact surfaces to delineate the articular cartilage in the hip joint. The geometry of the hip joint enabled creation of a radial distance map between the femoral and acetabular contact surfaces. We used a ray-firing method from the center of the femoral head to compute the radial distance from element centers of the acetabular surface to the femoral head contact surface (Figure 4). These radial distances comprised the cartilage thickness distribution over the acetabular surface.

\subsection{CONTACT PRESSURE COMPUTATION}

Contact pressure distributions over the articular surface were computed by DEA (An et al., 1990; Genda et al., 1995, 2001; Yoshida et al., 2006). The interaction of rigid bony structures through an elastic layer of cartilage was analyzed by modeling the cartilage layer as a set of compression springs over the potential contact area. Each triangular element of the acetabular surface mesh was modeled as a compression spring attached to the center of the

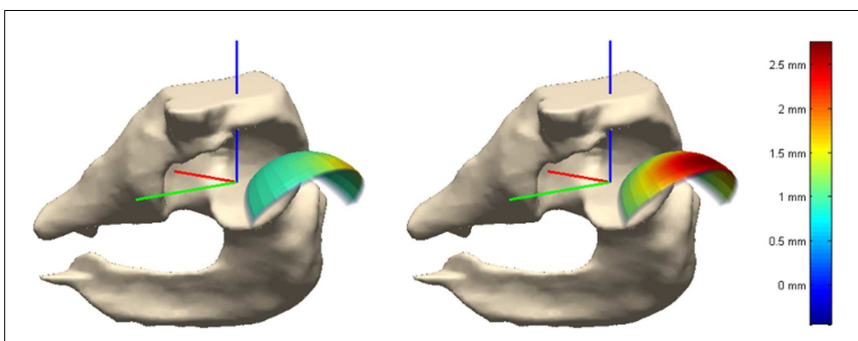

FIGURE 3 | Cartilage thickness map on weight-bearing area in normal (left) and dysplastic (right) populations. The weight-bearing area is moved out of the joint for better visualization.

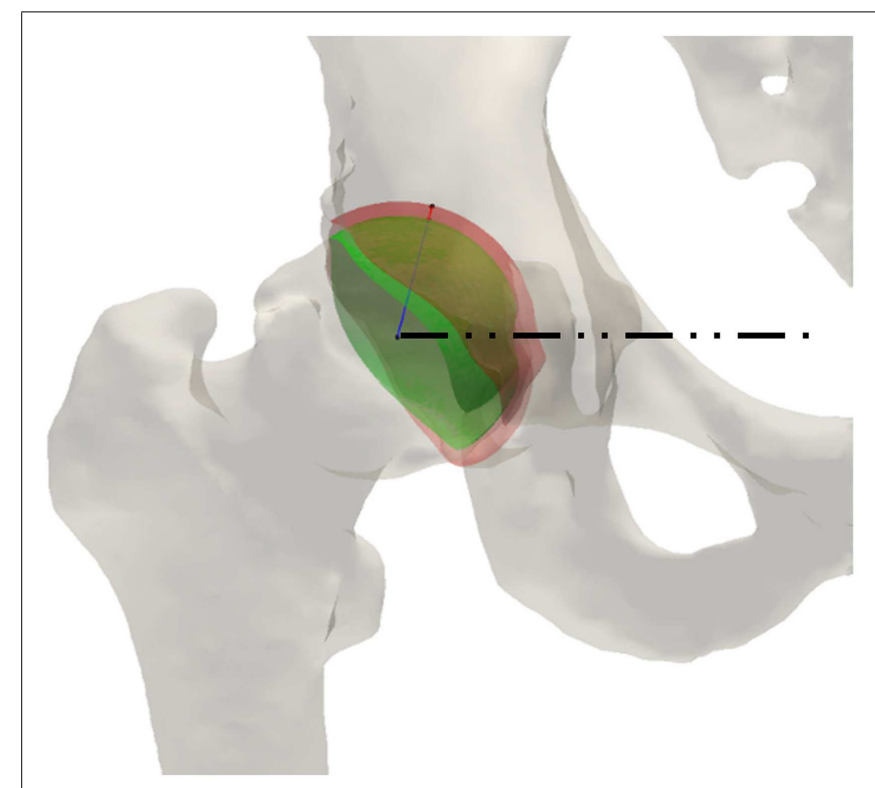

FIGURE 4 | Ray-firing method for indirect computation of cartilage thickness from CT data using bony surfaces. The green and red surface models represent the contact surface on femoral and acetabular sides, respectively. The blue line shows the radial direction from the center of the femoral head to an arbitrary point on the femoral head surface. The red line delineates the radial distance between the femoral head and acetabulum contact surfaces. The dashed line is the medio-lateral axis of the hip, extending through the centers of the femoral heads.

corresponding element. Since the elastic modulus of cartilage is much less than that of bone (e.g., Blankevoort et al., 1991), one can assume intra-element deformations are much smaller than interelement displacements, making DEA an appropriate method for contact pressure calculations (Kawai and Toi, 1981).

The classical formulation of DEA models cartilage as a set of linear compression springs (An et al., 1990). The shear spring constant $\left(k_{s}=0.001 \mathrm{~N} / \mathrm{mm}\right)$ was much less than the compressive spring constant $\left(k_{d}\right)$ to ensure negligible shear forces over the articular surface (Yoshida et al., 2006). The compressive spring constant $k_{d}$ was derived on a per-element basis as

$$
k_{d}=\frac{E(1-\nu) A_{i}}{(1+\nu)(1-2 \nu) h_{i}}
$$


assuming Young's modulus of $E=11.58 \mathrm{MPa}$ (Kempson, 1980; Yoshida et al., 2006), Poisson's ratio of $\nu=0.45$ (Blankevoort et al., 1991), $A_{i}$ the area of the specific element, and a $h_{i}$ the cartilage thickness of a specific element (Athanasiou et al., 1994). Linear DEA is widely used as a first approximation for calculating the contact pressure distribution in cartilage (An et al., 1990; Genda et al., 1995; Schuind et al., 1995; Fregly et al., 2003; Elias et al., 2004; Armiger et al., 2009). Volokh et al. (2007) previously investigated the limits of applicability of such a linear spring model and compared the non-linear axial stress-stretch law to experimental and analytical tests on one-dimensional confined compression of articular cartilage as reported by Ateshian et al. (1997) and Huang et al. (2005). Non-linear DEA inherently models the behavior of cartilage with greater detail and at a higher level of accuracy than classical, linear DEA (Volokh et al., 2007). Non-linear DEA models cartilage compression with non-linear springs to better match the stress-strain curve. Computationally, non-linear DEA employs a Taylor-series expansion of the Cauchy stress:

$$
\sigma(\varepsilon)=\frac{H_{A 0}\left((1+\varepsilon)^{2}-1\right)}{2(1+\varepsilon)^{2 \beta+1}} \exp \beta\left((1+\varepsilon)^{2}-1\right)
$$

about zero strain, $\varepsilon=0$, where $H_{A 0}$ and $\beta$ are material properties. In the present work, we used both linear and non-linear DEA to represent variations of cartilage compressibility.

\subsection{JOINT LOADING CONDITIONS}

The contact pressure profile over the articular surface was found while applying forces corresponding to daily activities of walking, sitting, and standing. The force pertaining to walking represents the peak of the reported forces over a gait cycle, while the sitting and standing forces denote steady state values. The activity forces - reported as percentage of body weight - were borrowed from previously published work on joint forces of patients with endoprostheses (Bergmann et al., 2001). A constant body weight of $74 \mathrm{~kg}$ (163 lbs) was assumed for all patients to remove any scaling effect of body weight on the absolute value of the contact pressure.

\subsection{OPTIMIZATION OF ACETABULAR SURFACE ORIENTATION}

The BGS computes the biomechanically optimal alignment of the acetabulum by searching the space of geometrically reasonable orientations of the contact surface (Armiger, 2006; Armiger et al., 2009; Murphy, 2010). The search space includes realignment rotations of $\pm 45^{\circ}$ in the sagittal plane, $\pm 60^{\circ}$ in the frontal plane, and $\pm 45^{\circ}$ in the axial plane. The optimal realignment transformation minimizes the sum of squared peak contact pressures computed for mechanical forces pertaining to daily activities of walking, sitting, and standing. An optimization routine based on a variant of the Levenberg-Marquardt algorithm for non-linear systems in the Matlab ${ }^{\circledR}$ Optimization Toolbox (The MathWorks, Inc. Natick, MA, USA) finds this optimal alignment. As this routine uses only peak contact pressures, the known underestimation and smooth pressure distribution obtained through DEA has no effect. We found the biomechanically optimal alignment of the acetabulum under combinations of different cartilage thickness profiles and models for variation of cartilage compressibility (modeled as a linear spring, linear DEA, or a non-linear spring, non-linear DEA).

\subsection{MEASURES OF VARIATION}

We found the biomechanically optimal alignment of the acetabulum for each patient using all possible combinations of the DEA technique (linear or non-linear) and cartilage thickness map (uniform, based on the normal or dysplastic population, and derived from CT). For each patient, we computed the peak contact pressure in the optimal alignments for the three daily activities (walking, sitting, and standing) and compared those with the preoperative peak contact pressure.

To characterize anatomical variations of the optimal alignments, we used the center-edge (CE) (Wiberg, 1939) and acetabular index (AC) (Tönnis, 1987) angles, which are commonly used radiological metrics for evaluation and surgical treatment of acetabular dysplasia (Figure 5). We evaluated the original and optimized alignments of the acetabulum in terms of satisfying the well-established acceptable ranges for these angles (Tallroth and Lepisto, 2006). We used the method of Armiger et al. (2007) to automatically determine these radiological angles for the original and optimal alignments of the acetabulum. We also compared the realignment rotations based on their rotation components in the different anatomical planes.

\subsection{STATISTICAL ANALYSIS}

Analysis of variance (ANOVA) was used to compare the relative decrease of peak pressure - from preoperative to optimal - in combinations with different cartilage thickness and compressive properties. In the event ANOVA testing exhibited significant differences among groups, we used Tukey's HSD (Honestly Significant Difference) test to determine which group(s) were significantly different. Two series of analyses were carried out to test for significant effects from the two factors: cartilage thickness map and compressive properties.

To study the effect of different cartilage thickness models, results with the same cartilage map but different DEA techniques were combined and the four groups were statistically compared. The influence of cartilage compressive properties was evaluated by combining all results from each DEA technique and comparing

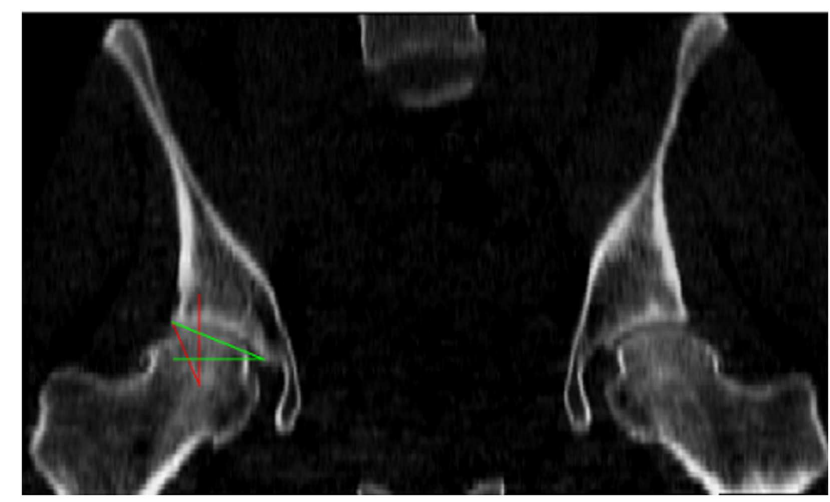

FIGURE 5 |The radiological angles of the hip include the center-edge (CE) angle, in red, which evaluates the lateral coverage of the femoral head by the acetabulum, and the acetabular index (AC) angle, in green, which represents the obliqueness of the acetabular roof 
the two groups (linear or non-linear DEA). We chose $p$-values smaller than 0.05 to indicate statistical significance.

\section{RESULTS}

The generated acetabular contact surfaces for each of the four algorithms were visually distinct (Figure 6). In the normal populationbased model, cartilage thickness values varied between 1.24 and $1.95 \mathrm{~mm}$; however, in the dysplastic population-based model, thickness values were increased and varied between 1.29 and $2.87 \mathrm{~mm}$. The increased cartilage thickness in the inferior portion of the acetabulum in the CT-based profile was likely an artifact of the ray-firing method. Since the inferior part of the acetabulum did not contribute to weight-bearing, these artifacts did not affect the contact pressure analysis.

In all cases, there was a relative decrease in peak contact pressure for all combinations of cartilage thickness distribution and compressive properties (Tables 1 and 2). On average, the optimal alignment decreased the peak contact pressure by $49.2 \pm 22.3 \%$. We found cartilage models to be significantly different from each other when comparing the relative decrease in peak pressure $(p<0.001)$. Specifically, the population-based dysplastic varied from the uniform and the CT-based, and the population-based normal varied from the CT-based. However, there was no significant difference between the linear and non-linear DEA techniques $(p=0.66)$.

In almost all patients, the value of maximum contact pressure exhibited a decrease from the original to the optimal alignment (Table 2); however, two patients exhibited a slight increase in the value of maximum pressure (less than $3 \%$ ) when optimal alignment was found using non-linear DEA with the population-based dysplastic cartilage distribution. On average, the CT-based cartilage thickness profile resulted in the largest decrease in peak contact pressures $(59 \pm 16 \%)$, followed by uniform $(52 \pm 21 \%)$ and population-based cartilage models $(47 \pm 22 \%$ for the normal population and $39 \pm 24 \%$ for the dysplastic population); i.e., CT-based cartilage models predicted alignments corresponding to the largest predicted benefit in alleviation of contact pressures. A sample pressure profile is illustrated in Figure 7 and a sample realignment is presented in Figure 8.

Different cartilage thickness models and DEA techniques resulted in similar improvements in radiological angles, all of which saw increased lateral coverage of the femoral head in the optimized location. Across the eight combinations of cartilage model and DEA technique, the average increase in CE angle from preoperative to optimal was $19.0 \pm 2.2^{\circ}$, ranging from $14.7^{\circ}$ to $21.5^{\circ}$ (Figure 9). Similarly, the average decrease in AC angle was $19.5 \pm 2.4^{\circ}$, ranging from $15.3^{\circ}$ to $22.4^{\circ}$ (Figure 10). Of the 22 cases with $\mathrm{CE}$ angle in the dysplastic range, $91.5 \%$ moved to normal range $\left(\mathrm{CE}>25^{\circ}\right)$ and $8.5 \%$ moved to borderline range $\left(20^{\circ}<\mathrm{CE}<25^{\circ}\right)$ after realignment.

\section{DISCUSSION}

Clinically, PAO is expected to alleviate pain associated with acetabular dysplasia by reducing joint contact pressure (Hipp et al., 1999; Leunig et al., 2001). Here, the relative decrease in maximum contact pressure from preoperative to optimal alignment was chosen as a metric describing how well a combination explains the

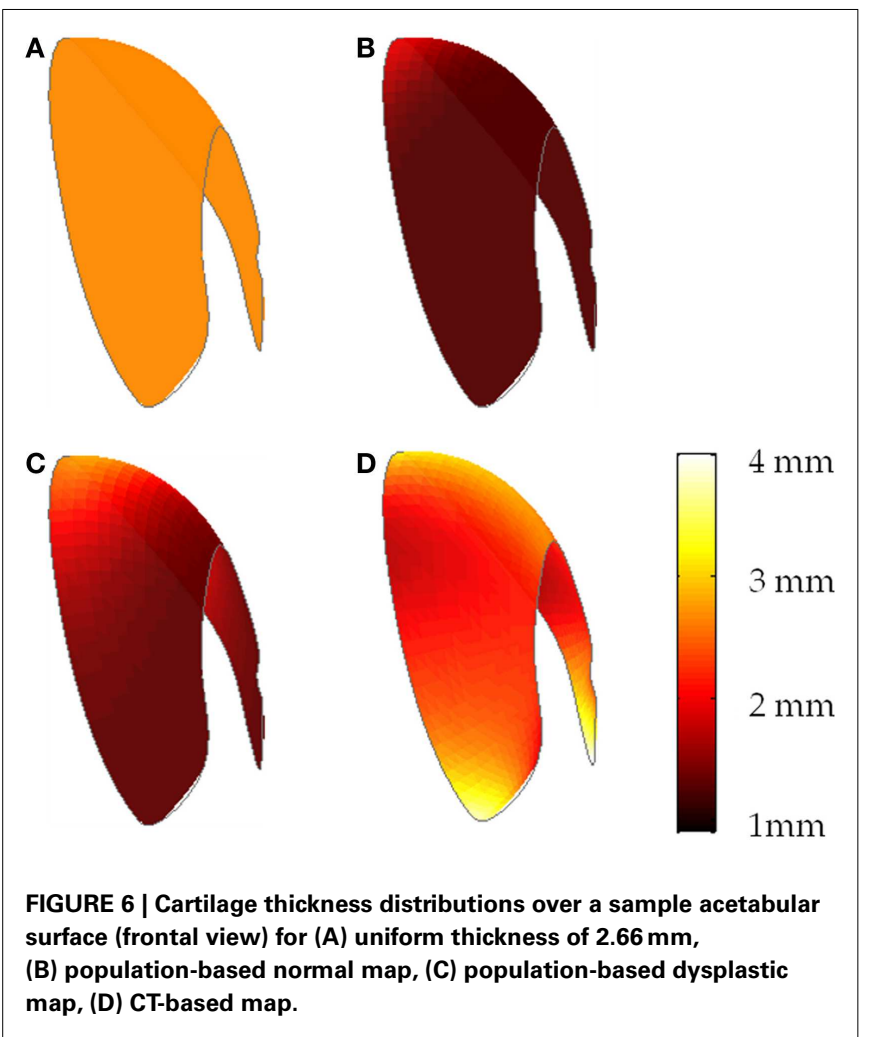

Table 1 | Relative decrease in peak contact pressure (\%) between preoperative and optimized alignments ("p-b" denotes a population-based model).

\begin{tabular}{llllll}
\hline $\begin{array}{l}\text { Calculation } \\
\text { method }\end{array}$ & \multicolumn{4}{c}{ Cartilage thickness model } & Mean \\
\cline { 2 - 5 } & Uniform & $\begin{array}{l}\text { p-b } \\
\text { Dysplastic }\end{array}$ & $\begin{array}{l}\text { p-b } \\
\text { Normal }\end{array}$ & $\begin{array}{l}\text { CT- } \\
\text { based }\end{array}$ \\
& & & & \\
\hline Linear DEA & $52 \pm 19$ & $42 \pm 22$ & $48 \pm 21$ & $55 \pm 16$ & $50 \pm 20$ \\
Non-linear DEA & $51 \pm 23$ & $35 \pm 24$ & $45 \pm 23$ & $62 \pm 16$ & $48 \pm 24$ \\
Mean & $52 \pm 21$ & $39 \pm 24$ & $47 \pm 22$ & $59 \pm 16$ & $49 \pm 22$ \\
\hline
\end{tabular}

effectiveness of acetabular realignment. In addition to the relative decrease in contact pressure, we studied the rotations which put the acetabular contact surface in the optimal alignment. We used these measures of variation to evaluate the role of cartilage thickness profile and DEA technique in the optimal alignment planning task in PAO.

As expected, all cartilage thickness distributions decreased the peak contact pressure from original to optimal alignment (Table 1). The CT-based cartilage distribution - which captures patient-specific cartilage geometry - predicted alignments resulting in the largest relative decrease in peak contact pressure. We speculate this may be due to the typical thickening of the cartilage seen at the lateral edge of the dysplastic acetabulum, which is no longer in the direct path of the load after realignment. 
Table 2 | Relative decrease in peak contact pressure data ("p-b" denotes a population-based model).

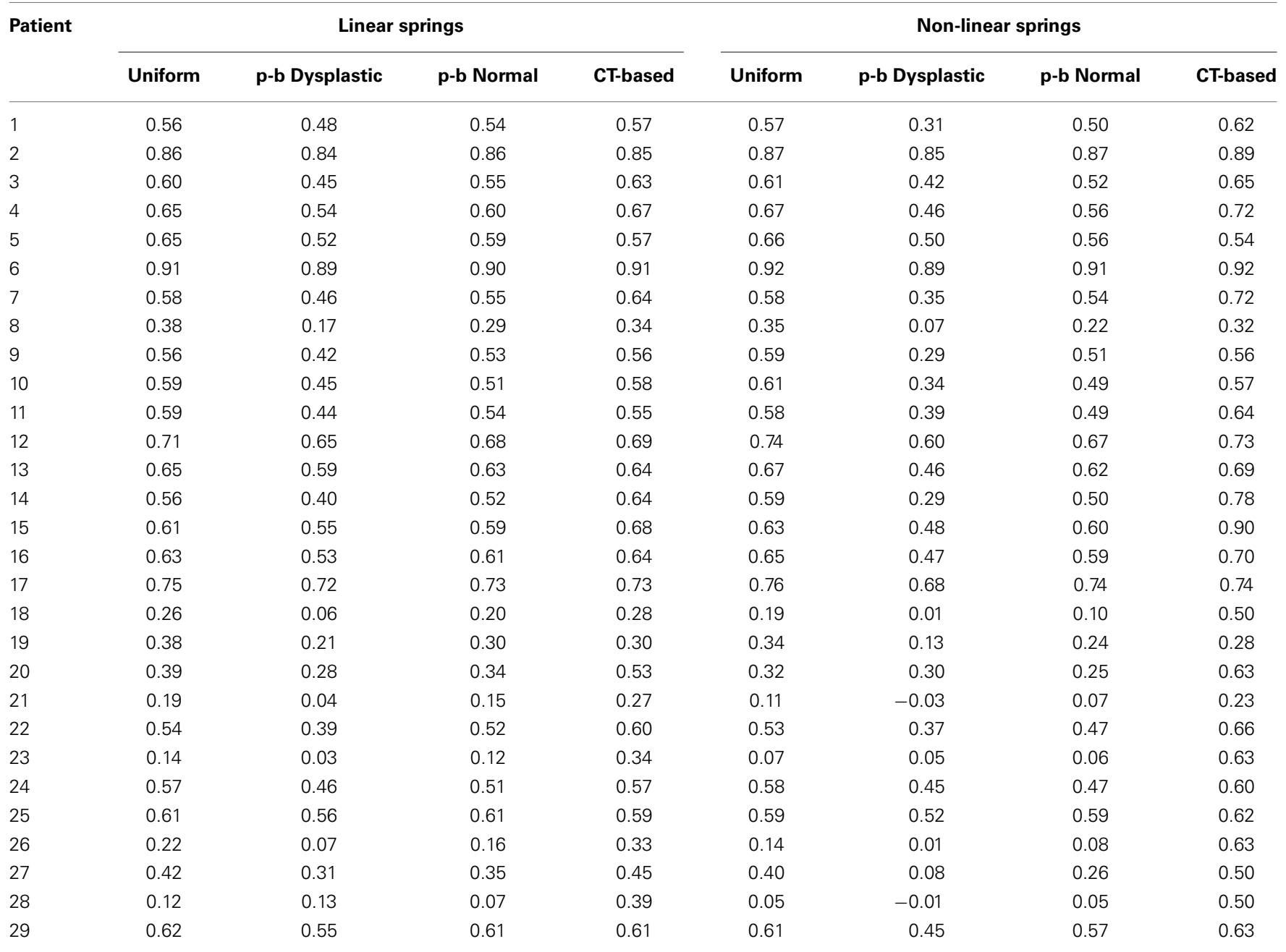

The optimal alignments from each thickness model showed, as expected, increased lateral coverage as defined by an increase in the $\mathrm{CE}$ angle and a decrease in the AC angle. However, the realignment rotations pertaining to the population-based dysplastic model increased the lateral coverage of the femoral head to a lesser degree compared to others. Of all 58 optimal alignments based on this thickness profile (with linear and non-linear DEA), 15 cases had $\mathrm{CE}$ angles below the normal range $\left(\mathrm{CE}<25^{\circ}\right)$. We speculate this may be due to the different thickness distribution in the dysplastic population; i.e., the abnormal acetabular configuration in dysplasia exposes the joint surfaces to elevated levels of contact pressure. This may elicit the body to provide more cartilage cushioning (i.e., greater cartilage thickness) over the weight-bearing area as a compensatory mechanism, which is consistent with the observations of Nishii et al. (2004). The thicker layer of articular cartilage will lower the contact pressure in dysplastic patients.

Our study has several limitations, including the technique to measure cartilage thickness on CT scans. CT arthrograms or MRI can be utilized to enhance contrast of the cartilaginous tissue (Chegini et al., 2009) and potentially increase the accuracy of the predicted optimal alignment of the acetabulum. However, the use of these modalities was not part of the existing clinical protocol for performing PAO at Orton Hospital. Moreover, the purpose of the study was to investigate if cartilage thickness would have a clinical impact on the biomechanically optimal alignment of the acetabulum. The results suggest that the optimal alignment was not significantly sensitive to the cartilage thickness profile.

Application of activity forces available from the literature (Bergmann et al., 2001) to the present joint models would yield more accurate results if the models were transformed to a consistent coordinate frame with the forces. Such transformations could not be defined for 19 subjects due to partial coverage of pelvis in their scan volumes. Therefore, forces were applied an intermediate coordinate frame. For 10 subjects where the entire pelvis scan was available, we compared two coordinate frames defined with or without the L5-S1 landmark. Among the 10 subjects, the adjusted supine frame differed from the accurate (Bergmann) coordinate frame by $8.0 \pm 4.6^{\circ}$. In the hips where we could define the Bergmann frame, there was a $0.5 \pm 0.35 \mathrm{MPa}$ difference in contact pressure between the supine and Bergmann frame. Moreover, a paired $t$-test between contact pressure in the Bergmann frame and those in the adjusted supine frame (that used in this study) 


\section{n}
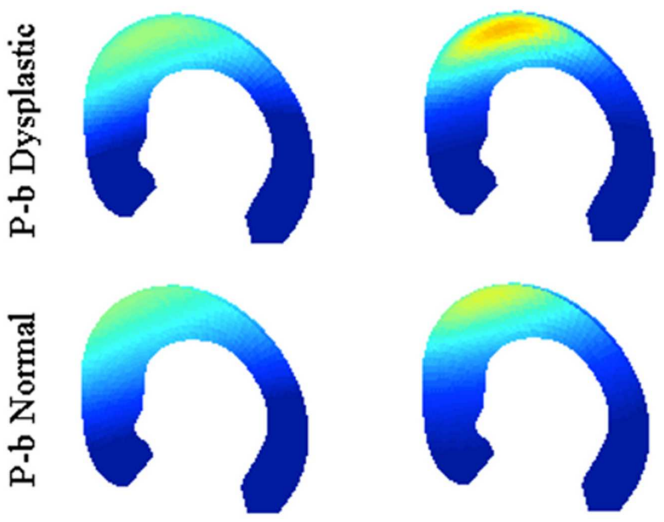

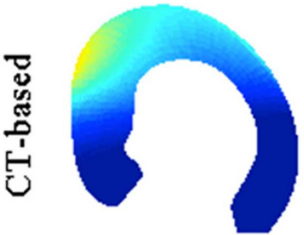

Linear DEA

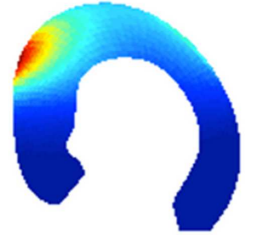

Nonlinear DEA
0.2
0.4
0.6
0.8
1.0
$1.2 \mathrm{MPa}$

FIGURE 7 | Pressure profile of a sample acetabulum loaded by the standing force (medial view). In this subject, the non-linear DEA-predicted higher stress levels over the acetabulum.

showed no significant difference between the data $(p=0.464)$. In addition, it is important to note that using the supine frame as an estimate of the standing frame is not an infrequent clinical practice and has also been used in radiological analysis of the hip (e.g., Genda et al., 2001; Armand et al., 2005b). We, therefore, believe our study makes good use of valuable, available data.

Studies as early as the one by Greenwald and Haynes (1972) have shown that, under normal stance loads, generally the entire articular surface of the acetabulum gets involved in bearing the load. Therefore, pressure is sometimes distributed over a relatively large area with more than one peak point similar to the results of Widmer et al. (2002). More detailed experiments and analyses show that, among the load-bearing areas, anterior and posterior aspects of the acetabulum see the peak pressure (Day et al., 1975; Sparks et al., 2005). Pressure patterns can sometimes resemble a band or a "ridge" rather than a simple peak, and in less frequent occasions can include double peaks (Brown and Shaw, 1983; Bay et al., 1997; von Eisenhart-Rothe et al., 1997; Brand et al., 2001). The observed pressure patterns are dependent on several factors
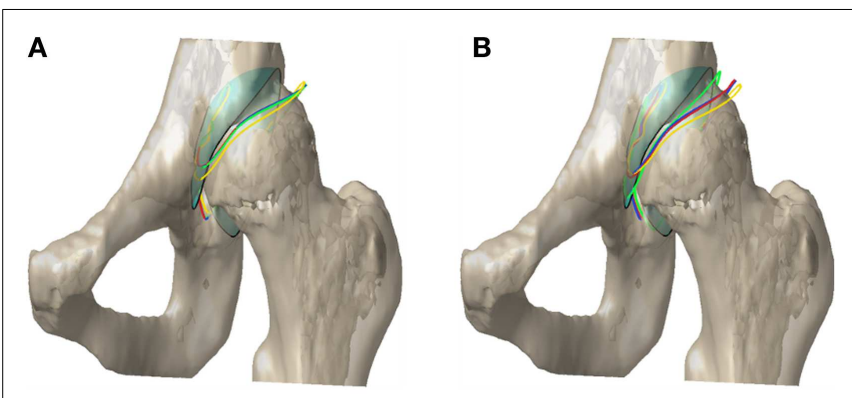

FIGURE 8 | Acetabular contact surface outline in the original and optimal alignment for (A) linear and (B) non-linear DEA. The colors red, blue, green, and yellow correspond to uniform, population-based ( $p-b)$ normal, p-b dysplastic, and CT-based cartilage thickness models, respectively. The cyan surface with the black outline represents the original alignment of the contact surface.

including the variations in the cartilage thickness, surface incongruity of the two contacting bones, and the amount and direction of loading (Bay et al., 1997; Sparks et al., 2005). The nature of our DEA analysis, similar to the model proposed by Yoshida et al. (2006), does not produce multiple peak pressure profiles. However, DEA achieves a first-order estimate of the pressure profile, which does include the critical superolateral pressure (near the edge of the weight-bearing zone) of interest when correcting dysplastic hip joints. Optimizing the orientation of the acetabular surface based on DEA-predicted pressures with single peaks (typically in the superolateral region) resulted in improvements in the radiological angles and the femoral head coverage. These improvements are compatible with the expected outcome of the surgery, encouraging the use of our DEA method for PAO optimization.

In conclusion, while patient-specific acetabular morphology is essential in planning PAO, the predicted optimal alignment of the acetabulum was not significantly sensitive to the choice of cartilage thickness distribution over the acetabulum. Moreover, each group showed clinical improvement in terms of decreasing contact pressures, increasing the lateral coverage, and decreasing the acetabular roof obliqueness. Therefore, through analysis of our patient-specific CT-based cartilage thickness profile, it appears that the additional cost and effort associated with the use of complex and non-uniform cartilage thickness profiles does not provide a great benefit for planning PAO. Moreover, the first-order estimation of the pressure profile using DEA does not appear to interfere with the clinical improvement seen when planning on peak pressure alone. However, future studies including more accurate MRI-based, patient-specific cartilage profiles can potentially present a more thorough argument. Furthermore, we investigated the effect of the constitutive model for cartilage compressibility. We found no significant difference between linear and non-linear DEA techniques by looking at the alleviation of contact pressures. However, similar to our observations regarding the cartilage thickness profiles, the rotational differences between linear and non-linear DEA techniques were less than seven degrees, minimizing their significance in clinical implications (i.e., surgical limitations in realization of the preoperative plan are likely to be a restricting factor on the precision). 


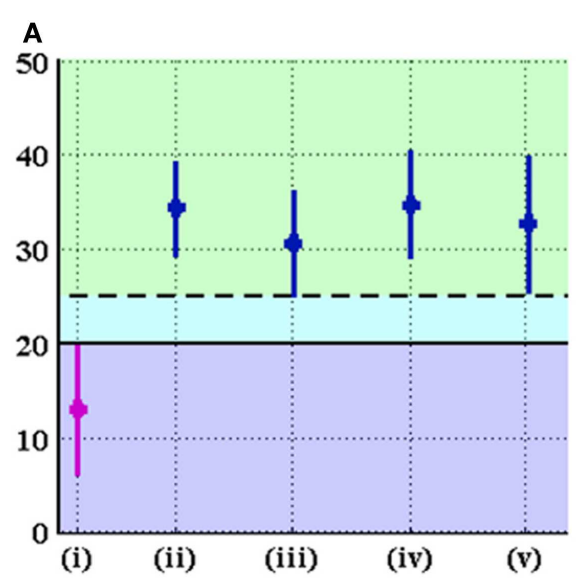

FIGURE 9 | CE angle (in degrees) in the original and optimal orientations of the acetabulum for (A) linear and (B) non-linear DEA. The graph displays mean \pm standard deviation of measurements for each group. The groups denoted by (i), (ii), (iii), (iv), and (v) represent the original alignment and the optimal alignments found using uniform, $p-b$ dysplastic, $p$-b normal, and

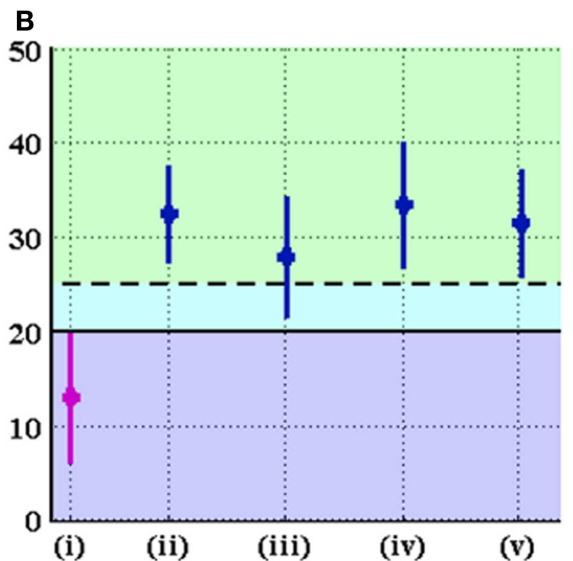

CT-based cartilage thickness models, respectively. The solid line marks the border between the dysplastic and borderline values of the CE angle, while the dashed line represents the border between borderline and normal values of the CE angle. Optimal orientation of the acetabulum results in improvement (increase) of CE angle.

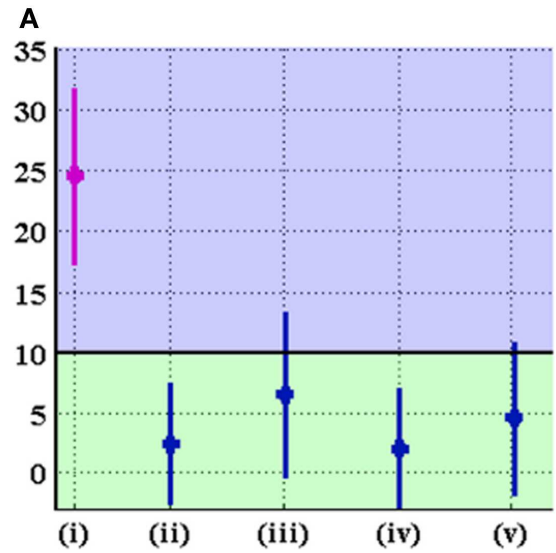

FIGURE 10 | AC angle (in degrees) in the original and optimal orientations of the acetabulum for (A) linear and (B) non-linear DEA. The graph displays mean \pm standard deviation of measurements for each group. The groups denoted by (i), (ii), (iii), (iv), and (v) represent the original alignment

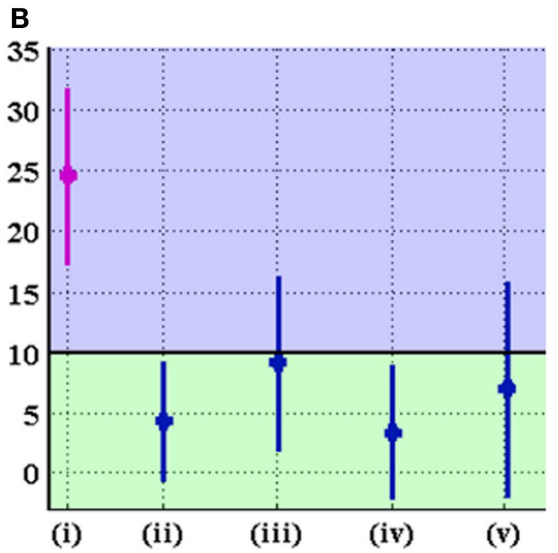

and the optimal alignments found using uniform, p-b dysplastic, p-b normal, and CT-based cartilage thickness models, respectively. The black line marks the border between normal and dysplastic values for the $\mathrm{AC}$ angle. Optimal orientation of the acetabulum results in improvement (decrease) of $\mathrm{AC}$ angle.

\section{ACKNOWLEDGMENTS}

We would like to thank Mr. Patrick Jongeneel for his work on evaluating the accuracy of the Lunate-Trace method. Funding: this work was supported by grant number R01 EB60389 from the National Institute for Biomedical Imaging and Bioengineering (NIH/NIBIB).

\section{REFERENCES}

Abraham, C. L., Maas, S. A., Weiss, J. A., Ellis, B. J., Peters, C. L., and Anderson, A. E. (2013). A new discrete element analysis method for predicting hip joint contact stresses. J. Biomech. 46, 1121-1127. doi:10.1016/j.jbiomech.2013.01.012

An, K., Himeno, S., Tsumura, H., Kawai, T., and Chao, E. (1990). Pressure distribution on articular surfaces: application to joint stability evaluation. J. Biomech. 23, 1013-1020. doi:10.1016/0021-9290(90)90316-U
Anda, S., Terjesen, T., Kvistad, K., and Svenningsen, S. (1991). Acetabular angles and femoral anteversion in dysplastic hips in adults: CT investigation. J. Comput. Assist. Tomogr. 15, 115-120. doi:10.1097/00004728199101000-00018

Anderson, A., Ellis, B., Maas, S., and Weissa, J. (2010). Effects of idealized joint geometry on finite element predictions of cartilage contact stresses in the hip. J. Biomech. 43, 1351-1357. doi:10.1016/j.jbiomech.2010.01.010

Anderson, A. E., Ellis, B. J., Maas, S. A., Peters, C. L., and Weiss, J. A. (2008). Validation of finite element predictions of cartilage contact pressure in the human hip joint. J. Biomech. Eng. 130, 051008. doi:10.1115/1.2953472

Armand, M., Armiger, R., Chintalapani, G., Liu, X., Szczepanowski, R., Feng, S., et al. (2005a). "Computer-assisted hip osteotomy surgery with real-time biomechanical feedback," in Annual Meeting of the International Society for Computer Assisted Orthopaedic Surgery (Helsinki: CAOS-International), 8-15.

Armand, M., Lepisto, J., Tallroth, K., Elias, J., and Chao, E. (2005b). Outcome of periacetabular osteotomy: joint contact pressure calculation using standing AP 
radiographs, 12 patients followed for average 2 years. Acta Orthop. 76, 303-313. doi:10.1080/00016470510030742

Armand, M., Lepisto, J., Merkle, A., Tallroth, K., Liu, X., Taylor, R., et al. (2004). Computer-aided orthopedic surgery with near-real-time biomechanical feedback. APL Tech. Dig. 25, 242-252.

Armiger, R. (2006). Development of a Surgical Guidance System for Real-Time Biomechanical Feedback during Periacetabular Osteotomy. Master's thesis, Johns Hopkins University, Baltimore, MD.

Armiger, R., Armand, M., Lepistö, J., Minhas, D., Tallroth, K., Mears, S., et al. (2007). Evaluation of a computerized measurement technique for joint alignment before and during periacetabular osteotomy. Comput. Aided Surg. 12, 215-224. doi:10.1080/10929080701541855

Armiger, R., Armand, M., Tallroth, K., Lepistö, J., and Mears, S. (2009). Threedimensional mechanical evaluation of joint contact pressure in 12 periacetabular osteotomy patients with 10-year follow-up. Acta Orthop. 80, 151-161. doi:10.3109/17453670902947390

Ateshian, G., Warden, W., Kim, J., Grelsamer, R., and Mow, V. (1997). Finite deformation biphasic material properties of bovine articular cartilage from confined compression experiments. J. Biomech. 30, 1157-1164. doi:10.1016/S00219290(97)85606-0

Athanasiou, K., Agarwal, A., and Dzida, F. (1994). Comparative study of the intrinsic mechanical properties of the human acetabular and femoral head cartilage. J. Orthop. Res. 12, 340-349. doi:10.1002/jor.1100120306

Bay, B. K., Hamel, A. J., Olson, S. A., and Sharkey, N. A. (1997). Statically equivalent load and support conditions produce different hip joint contact pressures and periacetabular strains. J. Biomech. 30, 193-196. doi:10.1016/S0021-9290(96) 00120-0

Bergmann, G., Deuretzbacher, G., Heller, M., Graichen, F., Rohlmann, A., Strauss, J., et al. (2001). Hip contact forces and gait patterns from routine activities. J. Biomech. 34, 859-871. doi:10.1016/S0021-9290(01)00040-9

Blankevoort, L., Kuiper, J., Huiskes, R., and Grootenboer, H. (1991). Articular contact in a three-dimensional model of the knee. J. Biomech. 24, 1019-1031. doi:10.1016/0021-9290(91)90019-J

Brand, R. A., Iglic, A., and Kralj-Iglic, V. (2001). Contact stresses in the human hip: implications for disease and treatment. Hip Int. 11, 117-126.

Brown, T. D., and Shaw, D. T. (1983). In vitro contact stress distributions in the natural human hip. J. Biomech. 16, 373-384. doi:10.1016/0021-9290(83) 90071-4

Chao, E., Armand, M., Nakamura, M., Matsuura, M., Genda, E., Lepistö, J., et al. (2000). Computer-Aided Hip Osteotomy Preoperative Planning, Vol. 25. Orlando, FL: Orthopaedic Research Society.

Chegini, S., Beck, M., and Ferguson, S. J. (2009). The effects of impingement and dysplasia on stress distributions in the hip joint during sitting and walking: a finite element analysis. J. Orthop. Res. 27, 195-201. doi:10.1002/jor.20747

Day, W., Swanson, S., and Freeman, M. (1975). Contact pressures in the loaded human cadaver hip. J. Bone Joint Surg. Br. 57, 302-313.

Elias, J., Wilson, D., Adamson, R., and Cosgarea, A. (2004). Evaluation of a computational model used to predict the patellofemoral contact pressure distribution. J. Biomech. 37, 295-302. doi:10.1016/S0021-9290(03)00306-3

Fregly, B., Bei, Y., and Sylvester, M. (2003). Experimental evaluation of an elastic foundation model to predict contact pressures in knee replacements. J. Biomech. 36, 1659-1668. doi:10.1016/S0021-9290(03)00176-3

Ganz, R., and Leunig, M. (2007). "Twenty years of experience with the Bernese periacetabular osteotomy for residual acetabular dysplasia," in Treatment of Osteoarthritic Change in the Hip, eds M. Sofue and N. Endo (Springer), 147-161.

Genda, E., Iwasaki, N., Li, G., MacWilliams, B., Barrance, P., and Chao, E. (2001). Normal hip joint contact pressure distribution in single-leg standing-effect of gender and anatomic parameters. J. Biomech. 34, 895-905. doi:10.1016/S00219290(01)00041-0

Genda, E., Konishi, N., Hasegawa, Y., and Miura, T. (1995). A computer simulation study of normal and abnormal hip joint contact pressure. Arch. Orthop. Trauma Surg. 114, 202-206. doi:10.1007/BF00444263

Greenwald, A., and Haynes, D. (1972). Weight-bearing areas in the human hip joint. J. Bone Joint Surg. Br. 54, 157-163.

Gu, D.-Y., Dai, K.-R., Hu, F., and Chen, Y.-Z. (2010). The shape of the acetabular cartilage surface and its role in hip joint contact stress. Conf. Proc. IEEE Eng Med. Biol. Soc. 2010, 3934-3937. doi:10.1109/IEMBS.2010.5627704
Hipp, J., Sugano, N., Millis, M., and Murphy, S. (1999). Planning acetabular redirection osteotomies based on joint contact pressures. Clin. Orthop. Relat. Res. 364, 134-143. doi:10.1097/00003086-199907000-00018

Hodler, J., Trudell, D., Pathria, M., and Resnick, D. (1992). Width of the articular cartilage of the hip: quantification by using fat-suppression spin-echo MR imaging in cadavers. AJR Am. J. Roentgenol. 159, 351-355. doi:10.2214/ajr.159.2. 1632354

Huang, C., Stankiewicz, A., Ateshian, G., and Mow, V. (2005). Anisotropy, inhomogeneity, and tension compression non-linearity of human glenohumeral cartilage in finite deformation. J. Biomech. 38, 799-809. doi:10.1016/j.jbiomech.2004. 05.006

Kawai, T., and Toi, Y. (1981). "A discrete method of limit analysis with simplified elements," in International Conference on Computing in Civil Engineering (New York: ASCE), 27-42.

Kempson, G. (1980). "The mechanical properties of articular cartilage," in The Joints and Synovial Fluid 2 (New York: Academic Press), 177-238.

Lenaerts, G., Bartels, W., Gelaude, F., Mulier, M., Spaepen, A., der Perre, G. V., et al. (2009). Subject-specific hip geometry and hip joint centre location affects calculated contact forces at the hip during gait. J. Biomech. 42, 1246-1251. doi:10.1016/j.jbiomech.2009.03.037

Lenaerts, G., Groote, F. D., Demeulenaere, B., Mulier, M., der Perre, G. V., Spaepen, A., et al. (2008). Subject-specific hip geometry affects predicted hip joint contact forces during gait. J. Biomech. 41, 1243-1252. doi:10.1016/j.jbiomech.2008.01. 014

Lepistö, J., Armand, M., and Armiger, R. (2008). Periacetabular osteotomy in adult hip dysplasia - developing a computer aided real-time biomechanical guiding system (BGS). Suom. Ortoped. Traumatol. 31, 186-190.

Leunig, M., Siebenrock, K., and Ganz, R. (2001). Rationale of periacetabular osteotomy and background work. Instr. Course Lect. 50, 229-238.

Li, G., Sakamoto, M., and Chao, E. (1997). A comparison of different methods in predicting static pressure distribution in articulating joints. J. Biomech. 30, 635-638. doi:10.1016/S0021-9290(97)00009-2

Mechlenburg, I., Nyengaard, J., Gelineck, J., Soballe, K., and Troelsen, A. (2010). Cartilage thickness in the hip measured by MRI and stereology before and after periacetabular osteotomy. Clin. Orthop. Relat. Res. 468, 1884-1890. doi:10.1007/ s11999-010-1310-z

Murphy, R. (2010). Development and Enhancement of Computer-Assisted Hip Surgeries for Periacetabular Osteotomy and Femoroacetabular Impingement. Master's thesis, Johns Hopkins University, Baltimore, MD.

Nishii, T., Sugano, N., Sato, Y., Tanaka, H., Miki, H., and Yoshikawa, H. (2004). Threedimensional distribution of acetabular cartilage thickness in patients with hip dysplasia: a fully automated computational analysis of MR imaging. Osteoarthr. Cartil. 12, 650-657. doi:10.1016/j.joca.2004.04.009

Nishii, T., Tanaka, H., Nakanishi, K., Sugano, N., Miki, H., and Yoshikawa, H. (2005). Fat-suppressed 3D spoiled gradient-echo MRI and MDCT arthrography of articular cartilage in patients with hip dysplasia. AJR Am. J. Roentgenol. 185, 379-385. doi:10.2214/ajr.185.2.01850379

Rushfeldta, P., Manna, R., and Harris, W. (1981). Improved techniques for measuring in vitro the geometry and pressure distribution in the human acetabulum. J. Biomech. 14, 253-260. doi:10.1016/0021-9290(81)90070-1

Schuind, F., Cooney, W., Linscheid, R., An, K., and Chao, E. (1995). Force and pressure transmission through the normal wrist. J. Biomech. 28, 587-601 doi:10.1016/0021-9290(94)00093-J

Siebenrock, K. A., Schöll, E., Lottenbach, M., and Ganz, R. (1999). Bernese periacetabular osteotomy. Clin. Orthop. Relat. Res. 363, 9-20.

Sparks, D. R., Beason, D. P., Etheridge, B. S., Alonso, J. E., and Eberhardt, A. W. (2005). Contact pressures in the flexed hip joint during lateral trochanteric loading. J. Orthop. Res. 23, 359-366. doi:10.1016/j.orthres.2004.08. 019

Tallroth, K., and Lepisto, J. (2006). Computed tomography measurement of acetabular dimensions: normal values for correction of dysplasia. Acta Orthop. 77, 598-602. doi:10.1080/17453670610012665

Tönnis, D. (1987). Congenital Dysplasia and Dislocation of the Hip in Children and Adults. Berlin: Springer.

Trumble, S. J., Mayo, K. A., and Mast, J. W. (1999). The periacetabular osteotomy. minimum 2 year followup in more than 100 hips. Clin. Orthop. Relat. Res. 363, $54-63$. 
Volokh, K., Chao, E., and Armand, M. (2007). On foundations of discrete element analysis of contact in diarthrodial joints. Mol. Cell. Biomech. 4, 67-73. doi:10.3970/mcb.2007.004.067

von Eisenhart-Rothe, R., Eckstein, F., Müller-Gerbl, M., Landgraf, J., Rock, C., and Putz, R. (1997). Direct comparison of contact areas, contact stress and subchondral mineralization in human hip joint specimens. Anat. Embryol. 195, 279-288. doi:10.1007/s004290050047

Wiberg, G. (1939). Studies on dysplastic acetabula and congenital subluxation of the hip joint with special reference to the complications of osteoarthritis. Acta Chir. Scand. 83, doi:10.1097/BPO.0b013e3182770a8d

Widmer, K.-H., Zurfluh, B., and Morscher, E. (2002). Load transfer and fixation mode of press-fit acetabular sockets. J. Arthroplasty 17, 926-935. doi:10.1054/ arth.2002.34526

Yoshida, H., Faust, A., Wilckens, J., Kitagawa, M., Fetto, J., and Chao, E. (2006). Threedimensional dynamic hip contact area and pressure distribution during activities of daily living. J. Biomech. 39, 1996-2004. doi:10.1016/j.jbiomech.2005.06.026

Zhao, X., Chosa, E., Totoribe, K., and Deng, G. (2010). Effect of periacetabular osteotomy for acetabular dysplasia clarified by three-dimensional finite element analysis. J. Orthop. Sci. 15, 632-640. doi:10.1007/s00776-010-1511-z
Conflict of Interest Statement: The authors declare that the research was conducted in the absence of any commercial or financial relationships that could be construed as a potential conflict of interest.

Received: 17 September 2013; paper pending published: 22 October 2013; accepted: 21 November 2013; published online: 10 December 2013.

Citation: Niknafs N, Murphy RJ, Armiger RS, Lepistö J and Armand M (2013) Biomechanical factors in planning of periacetabular osteotomy. Front. Bioeng. Biotechnol. 1:20. doi: 10.3389/fbioe.2013.00020

This article was submitted to Biomechanics, a section of the journal Frontiers in Bioengineering and Biotechnology.

Copyright (C) 2013 Niknafs, Murphy, Armiger, Lepistö and Armand. This is an open-access article distributed under the terms of the Creative Commons Attribution License (CC BY). The use, distribution or reproduction in other forums is permitted, provided the original author(s) or licensor are credited and that the original publication in this journal is cited, in accordance with accepted academic practice. No use, distribution or reproduction is permitted which does not comply with these terms. 